\title{
Cystadénome séreux bénin accompagné de toux et de lombalgie
}

\author{
Madeleine Rudolph BScS, Jessie Peng MD, Peter E. Wu MD MSc
}

— Citation : CMAJ 2021 August 23;193:E1304. doi : 10.1503/cmaj.202850-f

Voir la version anglaise de l'article ici : www.cmaj.ca/lookup/doi/10.1503/cmaj.202850

$\mathbf{U}$ ne femme de 27 ans s'est présentée au service des urgences en raison d'une toux sèche qui durait depuis 1 semaine et qui avait été précédée d'une lombalgie non traumatique depuis 1 mois. Sa fréquence cardiaque était de 110 battements/minute, et ses autres signes vitaux, son examen cardiaque et son examen respiratoire présentaient des résultats normaux. Elle avait un indice de masse corporelle élevé $\left(55 \mathrm{~kg} / \mathrm{m}^{2}\right)$ et ne présentait pas de masses palpables à l'examen abdominal. Sa formule sanguine complète montrait un taux d'hémoglobine de $86 \mathrm{~g} / \mathrm{L}$ (plage normale $120 \mathrm{~g} / \mathrm{L}-160 \mathrm{~g} / \mathrm{L}$ ), un

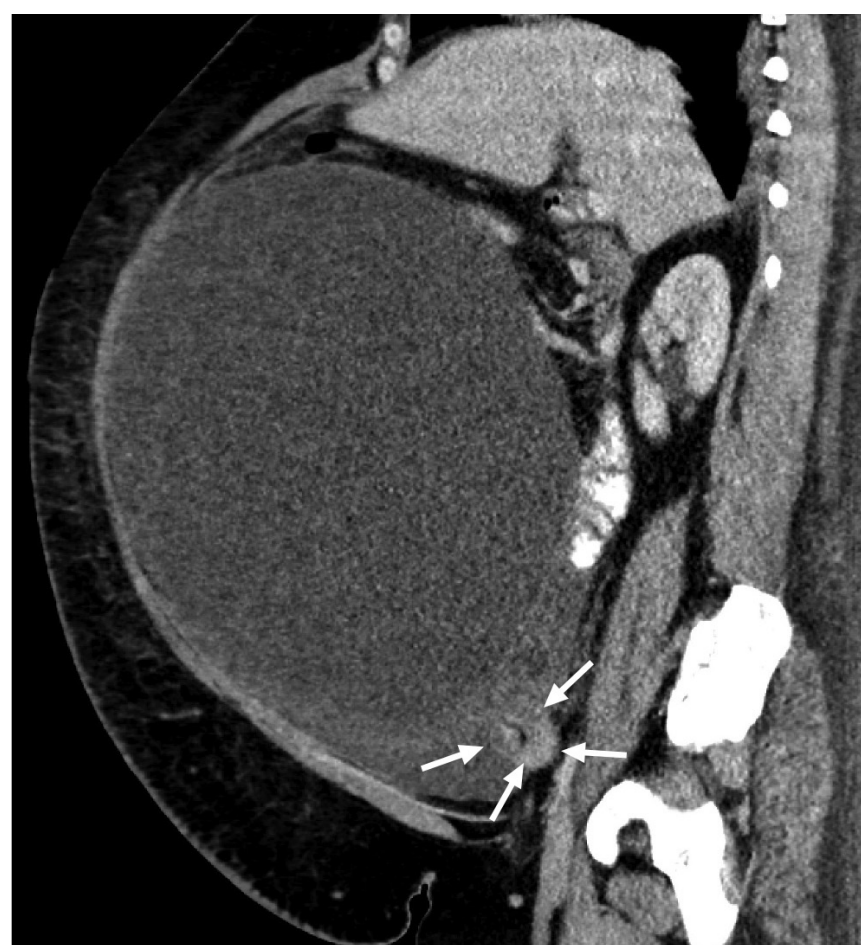

Figure 1 : Tomodensitométrie abdominale avec agent de contraste d'une femme de 27 ans ayant un cystadénome séreux. L'image en plan sagittal montre une masse de $34 \times 32,3 \times 25,7 \mathrm{~cm}$ et une torsade du pédicule ovarien droit (flèches), ainsi qu'un effet de masse et un déplacement supérieur du lobe gauche du foie. volume globulaire moyen de $85 \mathrm{fL}$ (plage normale $80 \mathrm{fL}-95 \mathrm{fL}$ ), un indice de distribution des globules rouges (coefficient de variation) de $15,7 \%$ (plage normale $11,5 \%-14,8 \%$ ), un taux d'éosinophiles de $0,6 \times 10^{9} / \mathrm{L}$ (plage normale $0,04 \times$ $10^{9} / \mathrm{L}-0,4 \times 10^{9} / \mathrm{L}$ ) et une numération plaquettaire de $801 \times 10^{9} / \mathrm{L}$ (plage normale $150 \times 10^{9} / \mathrm{L}-400 \times 10^{9} / \mathrm{L}$ ). Le test de gonadotrophine chorionique humaine ( $\beta$-hCG) présentait un résultat négatif. Un bilan poussé n'a permis de détecter aucune maladie auto-immune, maladie des tissus conjonctifs, infection ou exposition à des drogues ou médicaments. Pour déterminer la cause de la toux de la patiente, nous avons effectué une tomodensitométrie de son thorax, qui a montré des hyperdensités en verre dépoli non spécifiques et une atélectasie, ainsi que l'aspect supérieur d'une lésion intra-abdominale mal définie. Une tomodensitométrie de son abdomen (figure 1) a montré une importante masse kystique non marquée avec torsion conséquente, provenant de l'ovaire droit. Un dosage de l'antigène du cancer 125 (CA 125) a montré un taux de $355 \mathrm{U} / \mathrm{mL}$ (plage normale $\leq 34 \mathrm{U} / \mathrm{mL}$ ).

La patiente a subi en urgence une ovariectomie partielle et une salpingectomie. Nos chirurgiens ont excisé la masse kystique et drainé $11 \mathrm{~L}$ de liquide kystique teinté de sang. L'examen pathologique de la masse a donné un résultat négatif pour la malignité. Nous avons diagnostiqué un cystadénome séreux bénin avec infarctus et hémorragie secondaires dus à la torsion. Après la chirurgie, la toux, la lombalgie, l'éosinophilie, l'anémie et la thrombocytose, qu'on supposait réactifs, se sont résorbés chez la patiente.

Les cystadénomes séreux représentent environ $25 \%$ des tumeurs ovariennes bénignes chez les patientes en âge de procréer $^{1,2}$. Leur croissance est insidieuse, et le diagnostic peut être difficile à poser puisqu'ils sont souvent asymptomatiques $^{3}$. Les patientes ayant un cystadénome séreux ne ressentent souvent des symptômes que si la lésion subit une torsion ou exerce un effet de masse en raison de sa taille. C'était le cas chez notre patiente, dont la toux et la lombalgie l'ont poussée à consulter un médecin, ce qui a mené au diagnostic définitif et au traitement. 


\section{Références}

1. Glanc P, Benacerraf B, Bourne T, et al. First international consensus report on adnexal masses: management recommendations. J Ultrasound Med 2017;36:849-63.

2. Froyman W, Landolfo C, De Cock B, et al. Risk of complications in patients with conservatively managed ovarian tumours (IOTA5): a 2-year interim analysis of a multicentre, prospective, cohort study. Lancet Oncol 2019;20:448-58.

3. Salvador S, Scott S, Glanc $P$, et al. Guideline no. 403: initial investigation and management of adnexal masses. J Obstet Gynaecol Can 2020;42:1021-9.e3.

Intérêts concurrents : Aucun déclaré.

Cet article a été révisé par des pairs.

Les auteurs ont obtenu le consentement de la patiente.

Affiliations : Faculté de médecine (Rudolph) et Département de médecine (Peng, Wu), Université de Toronto, Toronto, Ont.

Propriété intellectuelle du contenu : Il s'agit d'un article en libre accès distribué conformément aux modalités de la licence Creative Commons Attribution (CC BY-NC-ND 4.0), qui permet l'utilisation, la diffusion et la reproduction dans tout médium à la condition que la publication originale soit adéquatement citée, que l'utilisation se fasse à des fins non commerciales (c.-à-d., recherche ou éducation) et qu'aucune modification ni adaptation n'y soit apportée. Voir : https://creativecommons.org/Licenses/by-nc-nd/4.0/deed.fr.

Remerciements : Les auteurs remercient le $D^{r}$ Marcus Bernardini pour ses commentaires et sa révision du manuscrit, le $D^{r}$ Luis Guimarães pour la révision et la mise en forme des images radiologiques, et ces deux médecins pour leurs soins à la patiente.

Correspondance : Madeleine Rudolph,m.rudolph@mail.utoronto.ca

Les images cliniques sont choisies pour leur caractère particulièrement intéressant, classique ou impressionnant. Toute soumission d'image de haute résolution claire et bien identifiée doit être accompagnée d'une légende aux fins de publication. On demande aussi une brève explication (300 mots maximum) de la portée éducative des images, et des références minimales. Le consentement écrit du patient au regard de la publication doit être obtenu avant la soumission. 